\title{
Cerebrospinal fluid amino acid concentrations in patients with viral and tuberculous meningitis
}

\author{
RN CORSTON, EHF MCGALE, C STONiER, EC HUTCHINSON, GM ABER
}

From the Department of Neurology, North Staffordshire Royal Infirmary and Renal Research Laboratory, Department of Post-Graduate Medicine, University of Keele, Staffordshire

SUMMARY Serial measurements have been made of the cerebrospinal fluid and plasma amino acid concentrations in 11 patients with viral meningitis and in four with tuberculous meningitis. A small increase in the concentrations of a few amino acids in cerebrospinal fluid has been found in a viral meningitis in contrast to a marked increase in most amino acids in tuberculous meningitis. The findings are compared with those found in patients with purulent meningitis and the possible causes and the diagnostic significance of these findings are discussed.

In normal individuals, mechanisms exist which maintain cerebrospinal fluid (CSF) concentrations of amino acids at a much lower level than those found in plasma. ${ }^{1}$ Minor disturbances of the CSF amino acid profile have been described in various neurological diseases including epilepsy, ${ }^{2}$ Parkinsonism and Huntington's chorea. ${ }^{3}{ }^{4}$ Marked changes have been described in patients with purulent meningitis, ${ }^{5}$ these changes resulting principally from a breakdown in the blood-CSF barrier to amino acids with lesser

Address for reprint requests: Dr RN Corston, Department of Neurology, Nottingham General Hospital, Nottingham NG1 6HA, UK.

Accepted 31 March 1981 contributions from damaged nervous tissue and white blood cells. In view of these observations, the present study was designed to establish whether any changes occur in the CSF amino acid composition in patients with either viral, or tuberculous meningitis.

\section{Patients and methods}

Eleven patients with viral meningitis and four with tuberculous meningitis were studied. Certain details of individual patients are given in Table 1. The diagnosis of meningitis was confirmed by microscopy, culture and chemical analysis of the lumbar CSF. Serological tests for viruses were carried out on individual patients when indicated. Subsequent

Table 1 Patients studied and details of laboratory investigations on initial CSF specimen

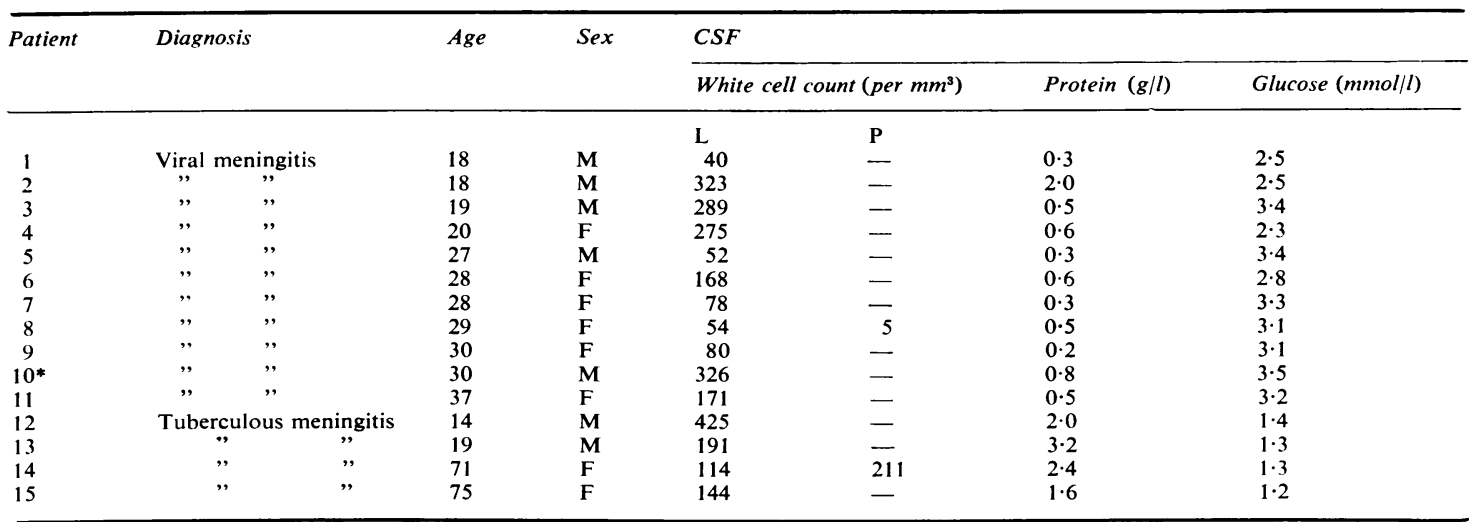

* Rise in antibody titre to Coxsachie virus

L: lymphocytes P: polymorphs 
examination of the CSF was carried out for clinical and therapeutic reasons. The diagnosis of viral meningitis was made on the basis of the clinical features and CSF findings consistent with this diagnosis. The diagnosis was confirmed by serological tests in one patient only (BW, Table 1). In all cases of tuberculous meningitis the diagnosis was confirmed by culture of Mycobacterium tuberculosis from the CSF. Drugs used in the treatment of tuberculous meningitis included streptomycin, isoniazid, PAS, ethambutol and rifampicin. On the basis of serial determinations of CSF amino acid concentrations before, during and after the use of these drugs, it would seem unlikely that these drugs have any direct effect on the CSF amino acid profile. The first clinical symptoms of meningitis (for example, headache, vomiting or impairment of consciousness) were taken as the starting point of the disease. All specimens, except those taken on hospital admission, were collected after a fast of 12-18 hours. Specimens of lumbar CSF and venous blood were centrifuged $\left(4000 \mathrm{~g}, 10 \mathrm{~min} 4^{\circ} \mathrm{C}\right)$ and deproteinised, stored and analysed for their free amino acid content as described previously. ${ }^{1}$ Glutamine and glutamic acid concentrations were measured on an aliquot of the same specimens by an enzymatic procedure. ${ }^{6}$ An aliquot of CSF was cultured and examined by microscopy. Quantitative measurements of protein and sugar concentrations and differential white cell count were carried out using standard laboratory procedures.

For statistical and temporal comparison of the pattern of amino acid change in the CSF, the results were divided into the following time periods.

(a) Tuberculous meningitis. Period 1 contains all values from the first day of clinical symptoms until day 30 of illness (mean 18.0 days). Period 2 contains all values between day 31 and 70 of illness (mean 46.0 days). Period 3 contains all values after day 70 of illness (mean 184.0 days). Values for CSF protein concentration, white cell count and plasma amino acid concentrations were grouped into the same time intervals.

(b) Viral meningitis. Period 1 contains all values from the first day of clinical symptoms to day 7 of illness (mean $5 \cdot 5$ days). Period 2 contains all values between day 8 and 15 of illness (mean 11.8 days). Period 3 contains all values after day 15 of illness (mean 38.0 days).

Results were compared with normal values ${ }^{2}$ using the Wilcoxon Rank Sum Test.

\section{Results}

CEREBROSPINAL FLUID

Changes in the total amino acid concentration, cell count and protein concentration are shown in Fig. 1. Since the data for tuberculous meningitis was obtained from four patients only, individual patient values are shown in Fig. 2. Individual CSF amino acid concentrations in patients with both viral and tuberculous meningitis at the time of maximum CSF abnormality are given in Table 2 . Full data can be obtained from the authors (RNC).
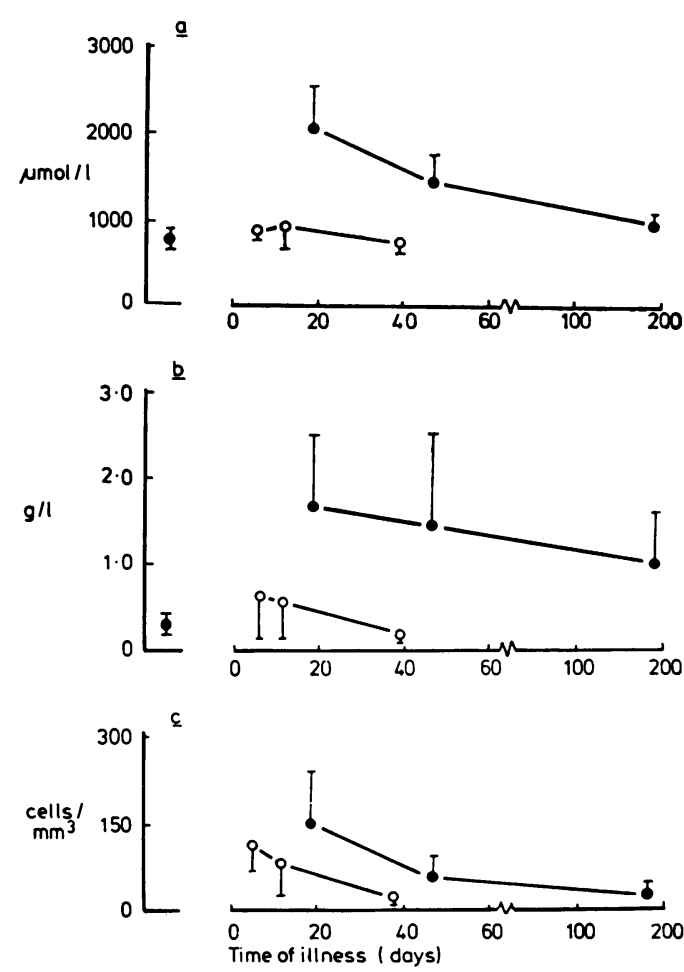

Fig 1 Comparison of serial changes in (a) total CSF amino acid concentration, $(b)$ protein, and $(c) C S F$ white cell count in tuberculous $(O)$ and viral $(O)$ meningitis with normal values (\$) All values are mean $\pm S D$ for the time periods defined in the text.

In viral meningitis, the total CSF amino acid concentration was increased abovenormal during the first 7 days of illness only $(924 \pm 91$ (SD) $\mu \mathrm{mol} / 1$, compared with $826 \pm 102 \mu \mathrm{mol} / \mathrm{l}$ in normals; $0.05>\mathrm{p}>0.02$ ). Examination of the data for individual CSF amino acid concentrations showed that eleven were present in increased concentration (Table 2) during the first period of illness only; thereafter, CSF concentrations of alanine and citrulline remained raised, all other values being within the normal range. The CSF concentrations of three amino acids (phosphoethanolamine, serine and glutamic acid) were reduced 


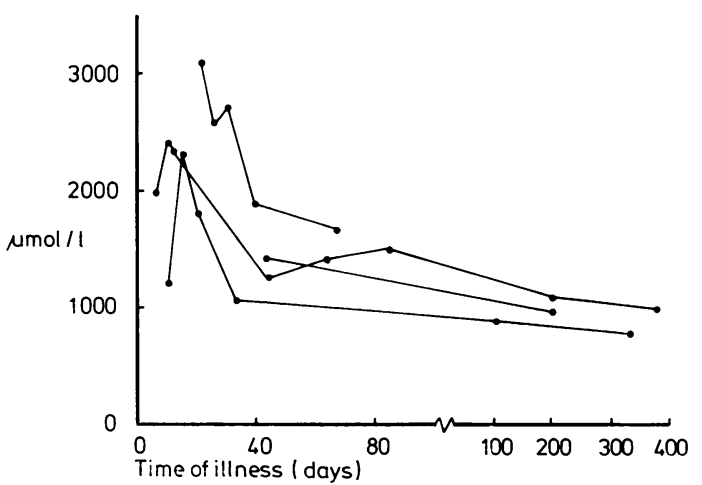

Fig. 2 Comparison of individual serial changes in total $C S F$ amino acid concentration in four patients with tuberculous meningitis.

during the third period of illness only.

In contrast, the quantitative and temporal pattern of change in CSF amino acids was markedly different in patients with tuberculous meningitis (fig 1). The total amino acid concentration was raised throughout all three periods of study, the maximum increase occurring in the first 0-30 days, when the mean total
CSF amino acid concentration was $2033 \pm 551 \mu \mathrm{mol} / 1$ compared with $826 \pm 102 \mu \mathrm{mol} / \mathrm{l}$ in normals $(p<0.001)$. Thereafter a gradual decrease to normal was noted. Examination of the data for individual amino acid concentrations showed that out of twentysix amino acids quantitated, the CSF concentrations of twenty-three were elevated during the first period of study, nineteen during the second period and sixteen during the third. Only one amino acid (serine) was present in normal concentration at all times of study. Glutamic acid concentration was reduced throughout the study.

\section{PLASMA}

In viral meningitis, the mean total plasma amino acid concentration was not different from normal at any time of study. In tuberculous meningitis, the mean total plasma amino acid concentration was reduced $(p<0.01)$ during the second period of study $(30-70$ days). Examination of the data for individual plasma amino acid concentrations showed that the plasma concentrations of 14 amino acids were reduced and three amino acid concentrations increased at various times of study. The plasma concentrations of phosphoserine, threonine and glutamine were reduced at all times of study.

Table 2 CSF amino acid and protein concentrations and white cell counts in normal individuals and in patients with viral and tuberculous meningitis at the time of maximum increase in total amino acid concentration. All values are $\mu \mathrm{mol} / \mathrm{l} \pm S D$

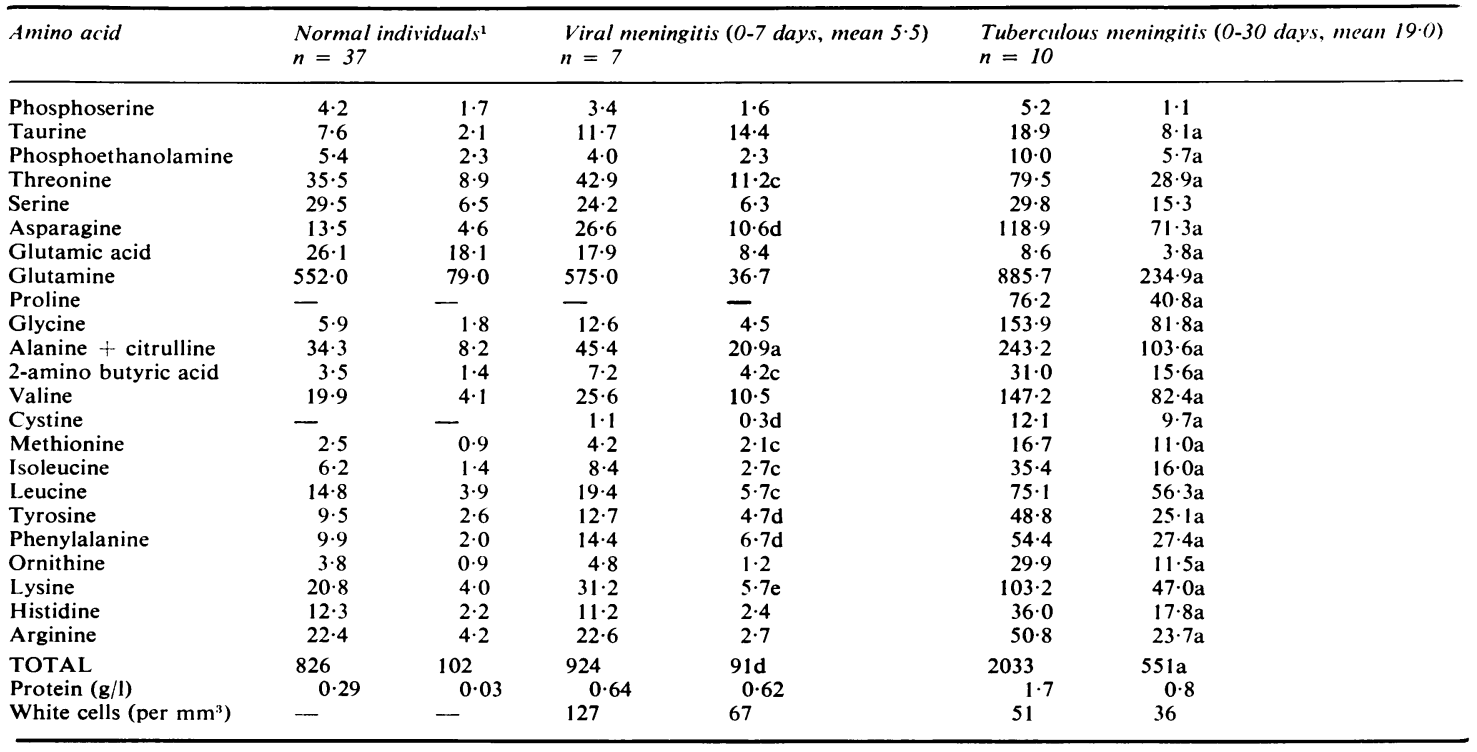

$\mathrm{a}=0.002>\mathrm{p}>0.001$

$\mathrm{b}=0.005>\mathrm{p}>0.001$

$c=0.02>p>0.01$

$\mathrm{d}=0.05>\mathrm{p}>0.02$

$\mathrm{e}=\mathrm{p}<0.01$

All other values not significantly different from normal $(p>0.05)$ 
CSF WHITE CELL COUNT

In both viral and tuberculous meningitis the total CSF white cell count was raised above normal in all patients throughout the study. Differential cell count showed that the increase was almost totally lymphocytic. The maximum values were found in the first period of the illness in both disorders. The mean maximum white cell count value for viral meningitis was 127 cells per $\mathrm{mm}^{3}$ ( $\left.\pm 67 \mathrm{SD}\right)$ and for tuberculous meningitis, 165 cells per $\mathrm{mm}^{3}( \pm 100 \mathrm{SD})$. The white cell counts subsequently fell progressively towards normal in both types of meningitis (fig 1).

\section{CSF PROTEIN}

In viral meningitis, the CSF protein concentration was raised during the first period of study in six of the eleven patients studied. The maximum protein concentration was present in the first 7 day period and was $0.64 \mathrm{~g} / 1$ ( $\pm 0.62 \mathrm{SD})$.

In tuberculous meningitis the CSF protein concentration was increased in all patients. The maximum increase occurred in the first period of the study and the mean concentration at this time was $1.73 \mathrm{~g} / 1$ (range $0 \cdot 55-3 \cdot 2 \mathrm{~g} / \mathrm{l}$ ).

In both viral and tuberculous meningitis, the CSF protein concentration subsequently fell progressively towards normal as shown in Fig. 1.

\section{Discussion}

The present study has shown that changes in the CSF amino acid composition occur in patients with viral and tuberculous meningitis. In patients with viral meningitis, there is a small increase in total amino acid concentration during the first few days of illness. Normal values were regained within a further week. In contrast, a large increase in total CSF amino acid concentration was found in patients with tuberculous meningitis, this occurring much later than was observed in patients with viral meningitis and the return to normal values was slower. Our findings contrast with those previously reported in purulent meningitis ${ }^{5}$ where a maximum increase was noted in the first week of illness, followed by a rapid return to normal values. The maximum mean values for total CSF amino acid concentration in viral, tuberculous and purulent meningitis were 1.2, 2.5 and 4.5 times normal respectively.

Examination of the data for changes in individual amino acid concentrations has yielded notable differences between viral and tuberculous meningitis which might have diagnostic significance. At the time of maximum abnormality in CSF amino acid composition, an increase in 11 amino acid concentrations was noted in viral meningitis, whereas in tuberculous meningitis, the CSF concentration of twenty-three amino acids was increased above normal. An increase in CSF glutamine concentration was found in purulent ${ }^{5}$ and tuberculous meningitis, but not in viral meningitis at any time of study. In contrast, the CSF concentration of glutamic acid was reduced in both viral and tuberculous meningitis but normal in patients with purulent meningitis. Both these metabolically related amino acids (glutamine and glutamic acid) play a major role in the metabolic activity of cerebral tissue and it is possible that their difference in behaviour may be related to the high affinity of cerebral tissue for glutamic acid. ${ }^{7}$ Since the clinical picture and CSF cellular content of viral and tuberculous meningitis may be similar during the early phase of illness, these differences may be of important diagnostic significance. Both these amino acids may be measured rapidly by a simple double-enzymatic procedure. ${ }^{6}$

There are several possible explanations of the changes in CSF amino acid composition observed during the present study. Firstly, alterations in the blood-CSF barrier to amino acid exchange to and from CSF. In support of this is the presence of significant quantities of proline and cystine in the CSF of patients with tuberculous meningitis. Both these amino acids are actively excluded from CSF in normal individuals and in patients with renal disease in spite of their presence in high concentrations in plasma. ${ }^{189}$ It seems reasonable to suggest that inflammatory changes in the choroid plexuses or meninges or both could produce disturbances in transport mechanisms similar to those noted in purulent meningitis. ${ }^{5}$ A less marked disturbance in amino acid transport across the blood-brain barrier in viral meningitis may be evidenced from a small increase in the CSF concentration of 11 amino acids only and an increase in the CSF concentration when compared with tuberculous meningitis. As in our previous study on purulent meningitis, ${ }^{5}$ none of the changes in CSF amino acids can be accounted for by a systematic disturbance of any of the known transport systems for amino acids within the choroid plexus. ${ }^{10}$

Secondly, changes in CSF glutamine in tuberculous meningitis might result from a disturbance in cerebral tissue metabolism, causing this amino acid to pass into the CSF, since glutamine is a major component of cerebral tissue. ${ }^{7}$ A similar increase in CSF glutamine concentration was noted in purulent meningitis. ${ }^{5}$ It is very unlikely that this increase in CSF glutamine concentration results from changes in plasma, since this is the only major amino acid component of both fluids where the CSF:plasma concentration ratio approaches unity in normal individuals ${ }^{1}$ and no increase in plasma concentration of this amino acid was found at the time of maximum 
CSF abnormality.

It is unlikely that the changes in CSF amino acid pattern described result from concomitant changes in plasma, since few changes in plasma composition were found. The possibility that these changes result from metabolism and destruction of white cells is also unlikely since there was no temporal correlation of amino acid and cellular change in viral, tuberculous or purulent meningitis and, further, in vitro studies involving study of the release of free amino acids from white cells incubated in CSF do not support this possibility (Corston, McGale, Hutchinson and Aber; unpublished observations).

We are grateful to the nursing staff in the Department of Neurology, North Staffordshire Royal Infirmary; and to Miss B Lawton for preparation of the manuscript. One of us (RNC) held a Sheldon Research Fellowship awarded by the West Midlands Regional Health Authority during the period of this study.

\section{References}

${ }^{1}$ McGale EHF, Pye IF, Stonier C, Aber GM, Hutchinson EC. Studies of the interrelationship between cerebrospinal fluid and plasma amino acid concentrations in normal individuals. J Neurochem 1977;29:291-7.
${ }^{2}$ Mutani R, Monaco F, Durelli L, Delsedieme M. The free amino acids in the cerebrospinal fluid of epileptic subjects. Epilephsia 1974;15:593-7.

${ }^{3}$ Lakke JRWF, Teelken AW. Amino acid abnormalities in the cerebrospinal fluid of patients with Parkinsonism and extrapyramidal disorders. Neurology (Minneap) 1976;26:489-93.

${ }^{4}$ Perry TL, Hansen S, Lesk D, Kloster M. Amino acids in plasma, cerebrospinal fluid and brain of patients with Huntington's chorea. Advances in Neurology 1973;1: 603-18.

5 Corston RN, McGale EHF, Stonier C, Hutchinson EC, Aber GM. Abnormalities of cerebrospinal fluid amino acids in purulent meningitis. $J$ Neurol Neurosurg Psychiatry 1979;42:881-6.

${ }^{6}$ Pye IF, Stonier C, McGale EHF. Double enzymatic assay for determination of glutamine and glutamic acid in cerebrospinal fluid and plasma. Anal Chem 1978;50: 951-3.

' Perry TL, Hansen S, Berry K, Mok C, Lesk D. Free amino acids and related compounds in biopsies of human brain. $J$ Neurochem $1971 ; 18: 521-8$.

${ }^{8}$ Pye IF, McGale EHF, Stonier C, Hutchinson EC, Aber GM. Studies on cerebrospinal fluid and plasma amino acids in patients with steady state chronic renal failure. Clin Chim Acta 1979;92:65-72.

${ }^{9}$ McGale EHF, Pye IF, Corston RN, Stonier C, Hutchinson EC, Aber GM. The effect of haemodialysis on cerebrospinal fluid and plasma amino acids. Clin Chim Acta $1978 ; 92: 73-80$

${ }^{10}$ Lorenzo AV. Amino acid transport mechanism of the cerebrospinal fluid. Federation Proc 1974;33:2079-85. 